\title{
Generalized Ait-Sahalia-type interest rate model with Poisson jumps and convergence of the numerical approximation
}

\author{
Shounian Deng ${ }^{\mathrm{a}, \mathrm{b}}$, Chen Fei ${ }^{\mathrm{c}}$, Weiyin Fei ${ }^{\mathrm{a}, *}$, Xuerong Mao ${ }^{\mathrm{d}}$ \\ ${ }^{a}$ The Key Laboratory of Advanced Perception and Intelligent Control of High-end Equipment, Ministry of Education, \\ and School of Mathematics and Physics, Anhui Polytechnic University, Wuhu, 241000, China. \\ ${ }^{b}$ School of Science, Nanjing University of Science and Technology, Nanjing, Jiangsu 210094, China \\ ${ }^{c}$ Glorious Sun School of Business and Management, Donghua University, Shanghai, 200051, China \\ ${ }^{d}$ Department of Mathematics and Statistics, University of Strathclyde, Glasgow G1 1XH,UK
}

\begin{abstract}
In this paper, we consider a generalized Ait-Sahalia interest rate model with Poisson jumps in finance. The analytical properties including positivity, boundedness and pathwise asymptotic estimations of the solution to this model are investigated. Moreover, we prove that the EulerMaruyama (EM) numerical solution converges to the true solution of the model in probability. Finally, we apply the EM solution to compute some financial quantities. A numerical example is provided to demonstrate the effectiveness of our results.
\end{abstract}

Keywords: Stochastic interest rate model, Poisson jumps, EM method, Convergence in probability.

\section{Introduction}

A classical problem in mathematical finance is the pricing of financial assets [1]. One of the asset pricing models reflecting the stochastic fluctuations is described by the following stochastic differential equation (SDE)

$$
d y(t)=\kappa(\mu-y(t)) d t+b y^{\theta} d B(t), \quad y(0)=y_{0},
$$

for any $t>0$. Here, $B(t)$ is a Brownian motion, $\kappa, \mu \geq 0, b, \theta>0$ and $y_{0}>0$. It is well known that (1.1) contains many famous models such as Merton [2], Vasicek [3], Cox et al. [4], and Brennan [5]. When $\theta=0.5,(1.1)$ is reduced to the mean-reverting square root model, namely, the CIR 5 model. In practice, successful applications of the asset pricing model are heavily dependent on the analytical properties (e.g., pathwise estimation, boundedness) of the true solution to the model. In general, stochastic asset pricing model has no explicit solution. Accordingly, there appear to be a practical need to estimate the true solution of the model via numerical approach. Thus the solution analysis and the convergence of the numerical approximation issues for the asset 10 pricing model have become hot topics of research attracting an ever-increasing interest. Higham

*Corresponding author

Email address: wyfei@ahpu.edu.cn (Weiyin Fei) 
and Mao [6] investigated the strong convergence properties of EM scheme for CIR model. Wu et al. [7] extended these results to the case of Poisson jumps. Dereich et al. [8] introduced a drift-implicit EM scheme which preserves positivity of solution. Hefter and Herzwurm [9] proposed a Milstein-type scheme and proved the strong convergence results. Analytical properties

15 of mean-reverting $\gamma$-model and the convergence result were obtained by Wu et al. [10], they also concentrated on the EM scheme for CIR model with delay [11]. The nonnegativity of solution to the mean-reverting-theta stochastic volatility model was proved by Baduraliya and Mao [12].

In [13] Ait-Sahalia studied several continuous-time models for interest rates. He tested parametric models by examining how closely the parametric model can reproduce features of its nonparametric counterpart. Results of the specification test suggested that a nonlinear stochastic interest model, which is now named as Ait-Sahalia-type model, captures well of the dynamics of the spot rate. Now Ait-Sahalia model has been widely used to volatility and other financial quantities besides interest rate [14, 15]. Furthermore, numerical method for Ait-Sahalia model also attracts researchers' attention. Cheng [16] discussed the analytical properties of this model

25 and showed that the EM solution converges to the true solution in probability. SSzpruch et al. [17] presented an implicit numerical method that preserves positivity and boundedness of moments and they proved the strong convergence result for the generalized Ait-Sahalia model. Results on the convergence of numerical solutions and ergodicity of this model can be found in Jiang et al. [18], Jin and Zhang [19].

30 However, the financial market is sensitive to unforeseen shocks and sudden events. Unexpected events may amplify uncertainty, which in turn exacerbates fear and increases risk perception in the financial system [20]. Numerous studies suggest strong empirical evidence that there exist jumps within financial markets and control systems, see e.g., Ait-Sahalia [21, 22, 23], Ma et al. [24], Li et al. [25]. Jumps risks cannot be ignored in the pricing of financial asserts (see

35 e.g., [26, 27]). As a more general jump-diffusion process, Lévy process has a wide range of applications in such diverse areas as mathematical finance, financial economics, and stochastic control [28]. For some recent works on Lévy noise, we refer the reader to the literature [28, 29, 30, 31, 32]. In order to characterize the impact of sudden and unforeseeable events on the term structure of interest rate and capture these discontinuous behaviors, jump-diffusion

40 processes have been applied in finance (see e.g., [33, 34, 35, 36, 37]).

Motivated by the above discussion, this paper is concerned with the generalized Ait-Sahalia interest rate model with Poisson jumps of the form

$$
\begin{aligned}
d y(t) & =\left(a_{-1} y^{-1}(t)-a_{0}+a_{1} y(t)-a_{2} y^{\gamma}(t)\right) d t+b y^{\theta}(t) d B(t)+\delta y\left(t^{-}\right) d N(t), \\
y(0) & =y_{0},
\end{aligned}
$$

for $t>0$. Here, $y_{0}, a_{-1}, a_{0}, a_{1}, a_{2}, b, \delta>0$ and $\theta, \gamma>1$. In addition, $y\left(t^{-}\right)=\lim _{s \rightarrow t^{-}} y(s), B(t)$ is a scalar Brownian motion and $N(t)$ is a scalar Poisson process with the compensated Poisson process $\tilde{N}(t)=N(t)-\lambda t$, where $\lambda$ denotes the jump intensity. Let $B(t)$ and $N(t)$ be independent We mainly focus on the analytical properties and numerical approximation of the true solution

45 to 1.2 . Threefold major contributions are highlighted as follows: (1) a novel generalized AitSahalia model is proposed to account for the phenomenon of Poisson jumps; (2) with the help of Lyapunov functions and stochastic analysis technique, the analytical properties including positivity, boundedeness and pathwise estimation of the solution to the model are obtained for the first time; (3) an appropriate numerical approach is constructed for solving the model and the corresponding convergence result is established. It is worth noticing that our results are not a straightforward generalization of Jiang et al. [18]. Some new techniques are developed to over- 
come the difficulties due to the appearance of Poisson jumps.

The rest of the paper is arranged as follows. In Section 2, we prove the nonnegativity of the solution to [1.2]. In Section 3, we discuss the boundedness of the true solution. Pathwise 55 estimations of the solution to (1.2) are investigated in Section 4. In Section 5, we show the convergence of the EM method applying to the model (1.2). Some applications are illustrated in Section 6. A numerical example together with some ideas on further research is presented in Section 7. Finally, we concludes this paper in Section 8.

\section{Positive and global solutions}

Throughout this paper, we assume that all the processes are defined on a complete probability space $(\Omega, \mathcal{F}, \mathbb{P})$ with a filtration $\left\{\mathcal{F}_{t}\right\}_{t \geq 0}$ satisfying the usual conditions (i.e., it is increasing and right continuous while $\mathcal{F}_{0}$ contains all $\mathbb{P}$-null sets). Let $x \vee y=\max (x, y)$ and $x \wedge y=\min (x, y)$ for any $x, y \in \mathbb{R}$. For a set $A, \mathbb{I}_{A}$ denotes its indicator function. Let $\lfloor u\rfloor$ denote the integer part of $u$, for any $u \in \mathbb{R}$. Moreover, we set $\inf \emptyset=\infty$. To obtain the desired results, we need the 65 following two lemmas.

Lemma 2.1. (The Itô formula with jumps [38]) Consider a jump-diffusion process

$$
y(t)=y(0)+\int_{0}^{t} f(y(s)) d s+\int_{0}^{t} g(y(s)) d B(s)+\int_{0}^{t} h\left(y\left(s^{-}\right)\right) d N(s), t>0 .
$$

Let $F(\cdot)$ be a twice continuously differential function. Then

$$
\begin{aligned}
F(y(t))= & F(y(0))+\int_{0}^{t}\left[F^{\prime}(y(s)) f(y(s))+\frac{1}{2} F^{\prime \prime}(y(s)) g^{2}(y(s))+\lambda(F(y(s)+h(y(s)))-F(y(s)))\right] d s \\
& +\int_{0}^{t} F^{\prime}(y(s)) g(y(s)) d B(s)+\int_{0}^{t}\left[F\left(y\left(s^{-}\right)+h\left(y\left(s^{-}\right)\right)\right)-F\left(y\left(s^{-}\right)\right)\right] d \widetilde{N}(t) .
\end{aligned}
$$

Lemma 2.2. Let $\mathbb{E} \int_{0}^{T}|h(s)|^{2} d s<\infty$. Then for any $T>0$

$$
\begin{aligned}
& \mathbb{E}\left|\int_{0}^{T} h\left(s^{-}\right) d N(s)\right|^{2} \leq 2 \lambda(1+\lambda T) \mathbb{E} \int_{0}^{T}|h(s)|^{2} d s, \\
& \mathbb{E}\left[\sup _{0 \leq t \leq T}\left|\int_{0}^{t} h\left(s^{-}\right) d \tilde{N}(s)\right|^{2}\right] \leq 4 \lambda \mathbb{E} \int_{0}^{T}|h(s)|^{2} d s, \\
& \mathbb{E}\left[\sup _{0 \leq t \leq T}\left|\int_{0}^{t} h\left(s^{-}\right) d N(s)\right|^{2}\right] \leq\left(8 \lambda+2 \lambda T^{2}\right) \mathbb{E} \int_{0}^{T}|h(s)|^{2} d s .
\end{aligned}
$$

The proof can be found in Fei et al. [39].

In the context of financial modeling, solution $y(t)$ denotes the asset price or the interest rate. It is essential to show that the solution is positive. The following theorem illustrates this property.

70 Theorem 2.3. For any given initial value $y_{0}>0$, there is a unique positive global solution $y(t)$ to 1.2 on $t \geq 0$. 
Proof. Let $\tau_{e}$ be the explosion time. For a sufficiently large positive integer $n$ satisfying $1 / n<$ $y(0)<n$, we define the stopping time

$$
\tau_{n}=\inf \left\{t \in\left[0, \tau_{e}\right): y(t) \notin[1 / n, n]\right\} .
$$

Noting that the coefficients of (1.2) are locally Lipschitz continuous, we can prove that there is a unique local solution $y(t) \in\left[0, \tau_{e}\right)$ for any given initial value $y_{0}>0$ by the classical methods [40]. Let $\tau_{\infty}=\lim _{n \rightarrow \infty} \tau_{n}$, which implies $\tau_{\infty} \leq \tau_{e}$, hence we need to show that $\tau_{\infty}=\infty$ a.s. , that 75 is $\lim _{n \rightarrow \infty} \mathbb{P}\left\{\tau_{n} \leq T\right\}=0$ for any $T>0$.

For any $0<\alpha<1$, we define a $C^{2}$-function $V:(0, \infty) \rightarrow(0, \infty)$ by

$$
V(y)=y^{\alpha}-1-\alpha \log y \text {. }
$$

It is easy to see that $V(y) \rightarrow \infty$ as $y \rightarrow \infty$ or $y \rightarrow 0$. We compute that

$$
V^{\prime}(y)=\alpha\left(y^{\alpha-1}-y^{-1}\right)
$$

and

$$
V^{\prime \prime}(y)=\alpha(\alpha-1) y^{\alpha-2}+\alpha y^{-2} .
$$

Hence,

$$
\begin{aligned}
& \mathbb{L} V(y)+\lambda(V(y+\delta y)-V(y)) \\
&=\alpha\left(y^{\alpha-1}-y^{-1}\right)\left(a_{-1} y^{-1}-a_{0}+a_{1} y-a_{2} y^{\gamma}\right)+\frac{b^{2}}{2}\left[\alpha(\alpha-1) y^{\alpha-2}+\alpha y^{-2}\right] x^{2 \theta} \\
&+\lambda\left[((1+\delta) y)^{\alpha}-1-\alpha \log ((1+\delta) y)-\left(y^{\alpha}-1-\log y\right)\right] \\
&= a_{-1} \alpha y^{\alpha-2}-a_{0} \alpha x^{\alpha-1}+a_{1} \alpha y^{\alpha}-a_{2} \alpha y^{\alpha+\gamma-1}-a_{-1} \alpha y^{-2}+a_{0} \alpha y^{-1} \\
&-a_{1} \alpha+a_{2} \alpha y^{\gamma-1}-\frac{b^{2} \alpha(1-\alpha)}{2} y^{\alpha+2 \theta-2}+\frac{b^{2} \alpha}{2} y^{2 \theta-2} \\
&+\lambda\left((1+\delta)^{\alpha}-1\right) y^{\alpha}-\lambda \alpha \log (1+\delta),
\end{aligned}
$$

where $\mathbb{L} V:(0, \infty) \rightarrow \mathbb{R}$ is defined by

$$
\mathbb{L} V(y)=V^{\prime}(y) f(y)+\frac{1}{2} V^{\prime \prime}(y) g^{2}(y),
$$

with $f(y)=a_{-1} y^{-1}-a_{0}+a_{1} y-a_{2} y^{\gamma}$ and $g(y)=b y^{\theta}$. Recalling that $0<\alpha<1, \gamma>1$ and $\theta>1$, we can deduce that $\mathbb{L} V(y)+\lambda(V(y+\delta y)-V(y))$ is bounded, say $K_{1}$, namely,

$$
\mathbb{L} V(y)+\lambda(V(y+\delta y)-V(y)) \leq K_{1}, \quad y \in(0, \infty) .
$$

By Lemma 2.1, for any $T>0$ we have

$$
\mathbb{E} V\left(y\left(T \wedge \tau_{n}\right)\right) \leq V\left(y_{0}\right)+K_{1} T .
$$

Therefore,

$$
\mathbb{P}\left(\tau_{n} \leq T\right)[V(1 / n) \wedge V(n)] \leq \mathbb{E} V\left(y\left(T \wedge \tau_{n}\right)\right) \leq V\left(y_{0}\right)+K_{1} T,
$$

which means

$$
\mathbb{P}\left(\tau_{n} \leq T\right) \leq \frac{V\left(y_{0}\right)+K_{1} T}{V(1 / n) \wedge V(n)} .
$$

Thus $\mathbb{P}\left(\tau_{n} \leq T\right) \rightarrow 0$, since $V(1 / n) \wedge V(n) \rightarrow \infty$ as $n \rightarrow \infty$. This means $\mathbb{P}\left(\tau_{\infty}=\infty\right)=1$ as required. 


\section{Boundedness}

In the modeling of stochastic interest rate, boundedness is a natural requirement. We establish so stochastic and moment boundedness for the solution to $[1.2]$ in this section.

\subsection{Boundedness of moments}

Theorem 3.1. For any $p \geq 2$, suppose that one of the following two conditions holds:

(i) $2 \theta<\gamma+1$

(ii) $2 \theta=\gamma+1$ and $a_{2}>(p-1) b^{2} / 2$

Then there is a constant $K_{2}$ such that the solution of (1.2) satisfies

$$
\mathbb{E} y^{p}(t) \leq \frac{y_{0}^{p}}{e^{t}}+K_{2}, \quad t>0 .
$$

Proof. For any $p \geq 2$, we define

$$
V_{1}(y, t)=e^{t} y^{p}, \quad(y, t) \in(0,+\infty) \times(0,+\infty) .
$$

Let $\tau_{n}$ be the stopping time defined in Theorem 2.3. We compute

$$
\begin{aligned}
\mathbb{L} & V_{1}(y, t)+\lambda\left(V_{1}(y+\delta y, t)-V_{1}(y, t)\right) \\
= & e^{t}\left[y^{p}+p y^{p-1}\left(a_{-1} y^{-1}-a_{0}+a_{1} y-a_{2} y^{\gamma}\right)+\frac{b^{2}}{2} p(p-1) y^{p-2+2 \theta}\right] \\
& +\lambda e^{t}\left((1+\delta)^{p}-1\right) y^{p} \\
= & e^{t}\left[p a_{-1} y^{p-2}-a_{0} p y^{p-1}+\left(a_{1}+1+\lambda\left((1+\delta)^{p}-1\right)\right) y^{p}\right. \\
& \left.-a_{2} p y^{p-1+\gamma}+\frac{b^{2}}{2} p(p-1) y^{p+2 \theta-2}\right] .
\end{aligned}
$$

In either condition (i) or condition (ii), we can deduce that there is a constant $K_{2}>0$ such that

$$
\mathbb{L} V_{1}(y, t)+\lambda\left(V_{1}(y+\delta y, t)-V_{1}(y, t)\right) \leq K_{2} e^{t} .
$$

Hence, for any $t \geq 0$,

$$
\mathbb{E}\left[e^{t \wedge \tau_{n}} y^{p}\left(t \wedge \tau_{n}\right)\right] \leq y_{0}^{p}+K_{2} e^{t} .
$$

Letting $n \rightarrow \infty$ and applying Fatou lemma, we obtain the desired assertion 3.1.

Theorem 3.2. For any $p \geq 1$, assume that $2 \theta \leq \gamma+1$ and $\gamma \leq p+1$. Then there is a constant $K_{3}$ such that the solution of (1.2) satisfies

$$
\mathbb{E} y^{-p}(t) \leq \frac{y_{0}^{-p}}{e^{t}}+K_{3}, \quad t>0 .
$$

Proof. Define

$$
V_{2}(y, t)=e^{t} y^{-p}, \quad(y, t) \in(0,+\infty) \times(0,+\infty) .
$$


Let $\tau_{n}$ be the same as before. We compute

$$
\begin{aligned}
& \mathbb{L} V_{2}(y, t)+\lambda\left(V_{2}(y+\delta y, t)-V_{2}(y, t)\right) \\
& =e^{t}\left[y^{-p}-p y^{-p-1}\left(a_{-1} y^{-1}-a_{0}+a_{1} y-a_{2} y^{\gamma}\right)+\frac{b^{2}}{2} p(p+1) y^{-p-2+2 \theta}\right] \\
& \quad+\lambda e^{t}\left((1+\delta)^{p}-1\right) y^{-p} .
\end{aligned}
$$

Recalling that $2 \theta \leq \gamma+1$ and $\gamma \leq p+1$, we can obtain that there is a constant $K_{3}>0$ such that

$$
\mathbb{L} V_{2}(y, t)+\lambda\left(V_{2}(y+\delta y, t)-V_{2}(y, t)\right) \leq K_{3} e^{t}
$$

Hence,

$$
\mathbb{E}\left[e^{t \wedge \tau_{n}} y^{-p}\left(t \wedge \tau_{n}\right)\right] \leq y_{0}^{-p}+K_{3} e^{t}
$$

Letting $n \rightarrow \infty$ and applying the Fatou lemma, we obtain the desired assertion (3.2).

By Theorem 3.1 and Theorem 3.2, we obtain the boundedness of $\mathbb{E} y^{2}(t)$ and $\mathbb{E} y^{-1}(t)$.

Corollary 3.3. Suppose that one of the following two conditions holds:

(i) $1<\theta \leq(\gamma+1) / 2$;

(ii) $\theta=(\gamma+1) / 2$ and $2 a_{2}>b^{2}$.

Then

$$
\mathbb{E} y^{2}(t)<\infty, \quad t>0 .
$$

Corollary 3.4. Suppose that $1<\theta \leq(\gamma+1) / 2$ and $1<\gamma \leq 2$. Then

$$
\mathbb{E} y^{-1}(t)<\infty, \quad t>0 .
$$

85 The following theorem shows that the average in time of the moments of the solutions $y(t)$ is bounded.

Theorem 3.5. For any $\alpha \in(0,1)$, there is a positive constant $K_{\alpha}$ such that for any initial value $y_{0}>0$, the solution of 1.2 satisfies

$$
\limsup _{t \rightarrow \infty}\left[\frac{1}{t} \int_{0}^{t} \mathbb{E} y^{\alpha+2 \theta-2}(s) d s\right] \leq K_{\alpha} .
$$

Proof. Noting that $\gamma>1$ and $\theta>1$. Then, there is a positive constant $K_{\alpha}$ such that

$$
\begin{aligned}
& \alpha\left(y^{\alpha-1}-y^{-1}\right)\left(a_{-1} y^{-1}-a_{0}+a_{1} y-a_{2} y^{\gamma}\right)+\frac{b^{2} \alpha}{2}\left(-\frac{1}{2}(1-\alpha) x^{\alpha+2(\theta-1)}+x^{2(\theta-1)}\right) \\
& +\lambda\left((1+\delta)^{\alpha}-1\right) y^{\alpha}-\lambda \alpha \log (1+\delta) \leq K_{\alpha} .
\end{aligned}
$$

Then we deduce from 2.1 that for any $t \in[0, T]$

$$
\frac{b^{2}}{4} \alpha(1-\alpha) \mathbb{E} \int_{0}^{t \wedge \tau_{n}} y^{\alpha+2 \theta-2}(s) d s+\mathbb{E} V\left(y\left(t \wedge \tau_{n}\right)\right) \leq V\left(y_{0}\right)+K_{\alpha} t .
$$

Letting $t \rightarrow \infty$ and using the Fatou lemma, we obatin

$$
\frac{b^{2}}{4} \alpha(1-\alpha) \mathbb{E} \int_{0}^{t} y^{\alpha+2 \theta-2}\left(s^{-}\right) d s \leq V\left(y_{0}\right)+K_{\alpha} t,
$$

which implies the required assertion. 
Theorem 3.6. If $\theta>1.5$, then there is a constant $K_{4}$ such that the solution of (1.2) satisfies

$$
\limsup _{t \rightarrow \infty} \frac{1}{t} \int_{0}^{t} \mathbb{E}\left(y^{-2}(s)+y^{2}(s)\right) d s \leq K_{4} .
$$

Proof. If $\theta>1.5$, we choose $\alpha \in(0.5,1)$, such that $\alpha+2(\theta-1)>2$. Hence, there is a constant $K_{4}$ such that

$$
\frac{1}{4} a_{-1}\left(y^{-2}+y^{2}\right)+\mathbb{L} V(y)+\lambda(V(y+\delta y)-V(y)) \leq \frac{1}{4} a_{-1} K_{4}, \quad y \in(0, \infty),
$$

where $\mathbb{L} V$ was defined in Theorem 2.3 . By 2.1 , we have

$$
\begin{aligned}
\frac{1}{4} \mathbb{E} \int_{0}^{t \wedge \tau_{n}}\left(y^{-2}(s)+y^{2}(s)\right) d s & \leq \frac{1}{4} \mathbb{E} \int_{0}^{t \wedge \tau_{n}}\left(y^{-2}(s)+y^{2}(s)\right) d s+\mathbb{E} V\left(y\left(t \wedge \tau_{n}\right)\right) \\
& \leq V\left(y_{0}\right)+\frac{1}{4} K_{4} t
\end{aligned}
$$

Hence, we get

$$
\frac{1}{t} \mathbb{E} \int_{0}^{t \wedge \tau_{n}}\left(y^{-2}(s)+y^{2}(s)\right) d s \leq \frac{4 V\left(y_{0}\right)}{t}+K_{4} .
$$

Letting $n \rightarrow \infty$ gives the desired assertion 3.3 .

\subsection{Stochastic boundedness}

90 In this subsection, we show that the solution of (1.2) stay within the interval $\left(1 / n_{1}, n_{1}\right)$ with a large probability for a positive constant $n_{1}$.

Theorem 3.7. For any $\varepsilon \in(0,1)$ and $y_{0}>0$, there is a constant $n_{1}=n_{1}(y(0), \varepsilon)>1$ such that the solution of 1.2 satisfies

$$
\mathbb{P}\left(1 / n_{1}<y(t)<n_{1}\right) \geq 1-\varepsilon . \quad t \geq 0 .
$$

Proof. Define

$$
V_{2}(y, t)=e^{t} V(y), \quad(y, t) \in(0,+\infty) \times(0,+\infty),
$$

where $V(y)=y^{\alpha}-1-\log y$. Let $\tau_{n}$ be the same as before. For any $t \in[0, T]$, Lemma2.1 gives

$$
\begin{aligned}
\left.\mathbb{E} V_{2}\left(y\left(t \wedge \tau_{n}\right), t \wedge \tau_{n}\right)\right)= & V\left(y_{0}\right)+\mathbb{E} \int_{0}^{t \wedge \tau_{n}} e^{s}[V(y(s))+\mathbb{L} V(y(s))] d s \\
& +\lambda \mathbb{E} \int_{0}^{t \wedge \tau_{n}} e^{s}\left[V\left(y\left(s^{-}\right)+\delta y\left(s^{-}\right)\right)-V\left(y\left(s^{-}\right)\right)\right] d s .
\end{aligned}
$$

By [2.1], there is a constant $K_{5}$ such that

$$
V(y)+\mathbb{L} V(y)+\lambda\left[V(y+\delta y)-V(y] \leq K_{5}, \quad y \in(0, \infty) .\right.
$$

Hence,

$$
\left.\mathbb{E} V_{2}\left(y\left(t \wedge \tau_{n}\right), t \wedge \tau_{n}\right)\right) \leq V\left(y_{0}\right)+K_{5} e^{t}
$$


Letting $n \rightarrow \infty$, we have

$$
e^{t} \mathbb{E} V(y(t)) \leq V\left(y_{0}\right)+K_{5} e^{t} .
$$

That is

$$
\mathbb{E} V(y(t)) \leq \frac{V\left(y_{0}\right)}{e^{t}}+K_{5} \leq V\left(y_{0}\right)+K_{5} .
$$

For any sufficiently large integer $n>1$, 3.6) gives

$$
\mathbb{P}(y(t) \leq 1 / n) \leq \mathbb{E}\left[\mathbb{I}_{\{y(t) \leq 1 / n\}} \frac{V(y(t))}{V(1 / n)}\right] \leq \frac{V\left(y_{0}\right)+K_{5}}{(1 / n)^{\alpha}-1-\alpha \log (1 / n)} \leq \frac{V\left(y_{0}\right)+K_{5}}{\alpha \log n-1} .
$$

Similarly,

$$
\mathbb{P}(y(t) \geq n) \leq \mathbb{E}\left[\mathbb{I}_{\{y(t) \geq n\}} \frac{V(y(t))}{V(n)}\right] \leq \frac{V\left(y_{0}\right)+K_{5}}{n^{\alpha}-1-\alpha \log n} .
$$

By 3.7) and 3.8, we have

$$
\mathbb{P}(1 / n<y(t)<n)>1-\left(V\left(y_{0}\right)+K_{5}\right)\left(\frac{1}{\alpha \log n-1}+\frac{1}{n^{\alpha}-1-\alpha \log n}\right) .
$$

Letting $n \rightarrow \infty$, we obtain the desired assertion.

\section{Pathwise asymptotic estimations}

We now begin to discuss the pathwise asymptotic properties of the true solution to 1.2 .

Theorem 4.1. Suppose that $1<\theta \leq 1.5$ and $1<\gamma \leq 2$. Then for any initial value $y_{0}>0$, the solution of $[1.2]$ satisfies

$$
\liminf _{t \rightarrow \infty} \frac{\log y(t)}{\log t} \geq-1 \quad \text { a.s. }
$$

Proof. Define $z(t)=y^{-1}(t)$. By Lemma 2.1, we have

$$
\begin{aligned}
d z(t)= & {\left[-a_{-1} z^{3}(t)+a_{0} z^{2}(t)-a_{1} z(t)+a_{2} z^{2-\gamma}(t)+b^{2} z^{3-2 \theta}(t)\right] d t } \\
& -b z^{2-\theta}(t) d B(t)-\frac{\delta}{1+\delta} z\left(t^{-}\right) d N(t) .
\end{aligned}
$$

Therefore, for $t>0$, we have

$$
\begin{aligned}
& \mathbb{E} z(t+1)+\frac{1}{2} a_{-1} \mathbb{E} \int_{t}^{t+1} z^{3}(s) d s \\
& =\mathbb{E} z(t)+\mathbb{E} \int_{t}^{t+1}\left[-\frac{a_{-1}}{2} z^{3}(s)+a_{0} z^{2}(s)-\left(a_{1}+\frac{\lambda \delta}{1+\delta}\right) z(s)+a_{2} z^{2-\gamma}(s)+b^{2} z^{3-2 \theta}(s)\right] d s .
\end{aligned}
$$

Recalling that $1<\theta \leq 1.5$ and $1<\gamma \leq 2$, we deduce that there are constants $K_{6}$ and $K_{7}$ such that

$$
-\frac{a_{-1}}{2} z^{3}+a_{0} z^{2}-\left(a_{1}+\frac{\lambda \delta}{1+\delta}\right) z+a_{2} z^{2-\gamma}+b^{2} z^{3-2 \theta} \leq K_{6}, \quad z \in(0,+\infty)
$$


and

$$
-a_{-1} z^{3}+a_{0} z^{2}-a_{1} z+a_{2} z^{2-r}+b^{2} z^{3-2 \theta} \leq K_{7}, \quad z \in(0,+\infty) .
$$

Hence, by 4.3 , we have

$$
\frac{1}{2} a_{-1} \mathbb{E} \int_{t}^{t+1} z^{3}(s) d s \leq \mathbb{E} z(t)+K_{6}
$$

For $u \in[t, t+1]$, 4.2] gives

$$
z(u) \leq z(t)+K_{7}-b \int_{t}^{u} z^{2-\theta}(s) d B(s)-\frac{\delta}{1+\delta} \int_{t}^{u} z\left(s^{-}\right) d N(s) .
$$

Applying the Lyapunov inequality, that is,

$$
\mathbb{E}|X|^{r} \leq\left(\mathbb{E}|X|^{s}\right)^{r / s}, \quad 0<r<s<\infty, \quad X \in L^{s}(\Omega),
$$

we have that

$$
\mathbb{E}\left[\int_{t}^{t+1} z^{2}(s) d s\right]^{1 / 2} \leq\left(\mathbb{E} \int_{t}^{t+1} z^{2}(s) d s\right)^{1 / 2} \leq\left(\left(\mathbb{E} \int_{t}^{t+1} z^{3}(s) d s\right)^{2 / 3}\right)^{1 / 2}=\left(\mathbb{E} \int_{t}^{t+1} z^{3}(s) d s\right)^{1 / 3},
$$

and

$$
\mathbb{E} \int_{t}^{t+1} z(s) d s \leq\left(\mathbb{E} \int_{t}^{t+1} z^{3}(s) d s\right)^{1 / 3} .
$$

Therefore, by the stochastic inequality, we have

$$
\begin{aligned}
\mathbb{E}\left[\sup _{t \leq u \leq t+1} \int_{t}^{u} z\left(s^{-}\right) d N(s)\right] & =\lambda \mathbb{E}\left[\sup _{t \leq u \leq t+1} \int_{t}^{u} z(s) d s\right]+\mathbb{E}\left[\sup _{t \leq u \leq t+1} \int_{t}^{u} z\left(s^{-}\right) d \tilde{N}(s)\right] \\
& \leq \lambda \mathbb{E} \int_{t}^{t+1} z(s) d s+C_{\lambda} \mathbb{E}\left[\int_{t}^{t+1} z^{2}(s) d s\right]^{1 / 2} \\
& \leq\left(\lambda+C_{\lambda}\right)\left(\mathbb{E} \int_{t}^{t+1} z^{3}(s) d s\right)^{1 / 3},
\end{aligned}
$$

where $C_{\lambda}$ is a constant. Similarly, using the Burkholder-Davis-Gundy inequality and the Lyapunov inequality gives

$$
\begin{aligned}
\mathbb{E}\left[\sup _{t \leq u \leq t+1} \int_{t}^{u} z^{2-\theta}(s) d B(s)\right] & \leq 3 \mathbb{E}\left[\int_{t}^{t+1} z^{2(2-\theta)}(s) d s\right]^{1 / 2} \\
& \leq 3\left(\mathbb{E} \int_{t}^{t+1} z^{2(2-\theta)}(s) d s\right)^{1 / 2} \\
& \leq 3\left(\mathbb{E} \int_{t}^{t+1} z^{3}(s) d s\right)^{(2-\theta) / 3} .
\end{aligned}
$$

$95 \quad$ By (4.4), 4.5) and 4.6, we get 


$$
\begin{aligned}
\mathbb{E}\left[\sup _{t \leq u \leq t+1} z(u)\right] & \leq \mathbb{E} z(t)+K_{7}+b \mathbb{E}\left[\sup _{t \leq u \leq t+1} \int_{t}^{u} z^{2-\theta}(s) d B(s)\right]+\frac{\delta}{1+\delta} \mathbb{E}\left[\sup _{t \leq u \leq t+1} \int_{t}^{u} z\left(s^{-}\right) d N(s)\right] \\
& \leq \mathbb{E} z(t)+K_{7}+3 b\left(\mathbb{E} \int_{t}^{t+1} z^{3}(s) d s\right)^{(2-\theta) / 3}+\frac{\delta\left(\lambda+C_{\lambda}\right)}{1+\delta}\left(\mathbb{E} \int_{t}^{t+1} z^{3}(s) d s\right)^{1 / 3} .
\end{aligned}
$$

By the boundedness of $\mathbb{E} z(t)$ and $\mathbb{E} \int_{t}^{t+1} z^{3}(s) d s$, we deduce that there is a constant $K_{8}$ such that

$$
\mathbb{E}\left[\sup _{t \leq u \leq t+1} z(u)\right] \leq K_{8}
$$

Let $\varepsilon>0$ be arbitrary. Applying the Chebyshev inequality gives

$$
\mathbb{P}\left(\sup _{n \leq t \leq n+1} z(t)>n^{1+\varepsilon}\right) \leq \frac{K_{8}}{n^{1+\varepsilon}}, \quad n=1,2, \cdots .
$$

By the Borel-Cantelli lemma, we have that for almost $\omega \in \Omega$, there is a $n_{0}(\omega)$ such that

$$
\sup _{n \leq t \leq n+1} z(t) \leq n^{1+\varepsilon} \text {, for } n \geq n_{0}, n \leq t \leq n+1
$$

which means

$$
\frac{\log z(t)}{\log t} \leq \frac{(1+\varepsilon) \log n}{\log n}=1+\varepsilon
$$

That is

$$
\liminf _{t \rightarrow \infty} \frac{\log y(t)}{\log t} \geq-(1+\varepsilon)
$$

Letting $\varepsilon \rightarrow 0$, we obtain the desired assertion (4.9). Thus, the proof is complete.

Theorem 4.2. Suppose that $1<\theta<(\gamma+1) / 2$ and $1<\gamma \leq 2$. Then for any initial value $y(0)>0$, the solution of 1.2 satisfies

$$
\limsup _{t \rightarrow \infty} \frac{\log y(t)}{\log t} \leq 1 \quad \text { a.s. }
$$

Proof. By Lemma 2.1, we have

$$
\begin{aligned}
d\left[y^{2}(t)\right]= & {\left[2 y(t)\left(a_{-1} y^{-1}(t)-a_{0}+a_{1} y(t)-a_{2} y^{\gamma}(t)\right)+b^{2} y^{2 \theta}(t)\right] d t } \\
& +2 b y^{\theta+1}(t) d B(t)+\left(2 \delta+\delta^{2}\right) y^{2}\left(t^{-}\right) d N(t) \\
= & {\left[2 a_{-1}-2 a_{0} y(t)+2 a_{1} y^{2}(t)-2 a_{2} y^{\gamma+1}(t)+b^{2} y^{2 \theta}(t)\right] d t } \\
& +2 b y^{\theta+1}(t) d B(t)+\left(2 \delta+\delta^{2}\right) y^{2}\left(t^{-}\right) d N(t) .
\end{aligned}
$$

Recalling that $1<\theta<(\gamma+1) / 2$ and $1<\gamma \leq 2$. Let $\eta \in\left(0,2 a_{2}\right)$ be arbitrarily small. Then there is a constant $K_{9}>0$ such that

$$
\eta y^{\gamma+1}+\left[2 a_{-1}-2 a_{0} y+2 a_{1} \lambda\left(2 \delta+\delta^{2}\right) y^{2}-2 a_{2} y^{\gamma+1}+b^{2} y^{2 \theta}\right] \leq K_{9}, \quad y \in(0, \infty) .
$$


By 4.10, 4.11) and Corollary 3.3, we have

$$
\begin{aligned}
\eta \mathbb{E} \int_{t}^{t+1} y^{\gamma+1}(s) d s & \leq \eta \mathbb{E} \int_{t}^{t+1} y^{\gamma+1}(s) d s+\mathbb{E} y^{2}(t+1) \\
& \leq \mathbb{E} y^{2}(t)+K_{9}<\infty
\end{aligned}
$$

for any $t \in[0, T]$. Then for any $u \in[t, t+1]$, we have

$$
\begin{aligned}
y(u)= & y(t)+\int_{t}^{u}\left[a_{-1} y^{-1}(s)-a_{0}+a_{1} y(s)-a_{2} y^{\gamma}(s)\right] d s \\
& +\int_{t}^{u} b y^{\theta}(s) d B(s)+\int_{t}^{u} \delta y\left(s^{-}\right) d N(s) .
\end{aligned}
$$

There is a constant $K_{10}$ such that

$$
-a_{0}+a_{1} y-a_{2} y^{\gamma} \leq K_{10}, \quad y \in(0,+\infty) .
$$

Hence

$$
y(u) \leq y(t)+K_{10}+a_{-1} \int_{t}^{u} y^{-1}(s) d s+\int_{t}^{u} b y^{\theta}(s) d B(s)+\int_{t}^{u} \delta y\left(s^{-}\right) d N(s),
$$

which implies

$$
\begin{aligned}
\mathbb{E}\left[\sup _{t \leq u \leq t+1} y(u)\right] \leq & \mathbb{E} y(t)+K_{10}+a_{-1} \int_{t}^{t+1} \mathbb{E} y^{-1}(s) d s \\
& +b \mathbb{E}\left[\sup _{t \leq u \leq t+1} \int_{t}^{u} y^{\theta}(s) d B(s)\right]+\delta \mathbb{E}\left[\sup _{t \leq u \leq t+1} \int_{t}^{u} y\left(s^{-}\right) d N(s)\right] .
\end{aligned}
$$

By the Burkholder-Davis-Gundy inequality and the Lyapunov inequality, we have

$$
\begin{aligned}
\mathbb{E}\left[\sup _{t \leq u \leq t+1} \int_{t}^{u} y^{\theta}(s) d B(s)\right] & \leq 3 \mathbb{E}\left[\int_{t}^{t+1} y^{2 \theta}(s) d s\right]^{1 / 2} \\
& \leq 3\left(\mathbb{E} \int_{t}^{t+1} y^{2 \theta}(s) d s\right)^{1 / 2} \\
& \leq 3\left(\mathbb{E} \int_{t}^{t+1} y^{\gamma+1}(s) d s\right)^{\theta /(\gamma+1)} .
\end{aligned}
$$

In the same way as 4.5 was obtained, we also have

$$
\begin{aligned}
\mathbb{E}\left[\sup _{t \leq u \leq t+1} \int_{t}^{u} y\left(s^{-}\right) d N(s)\right] & =\lambda \mathbb{E}\left[\sup _{t \leq u \leq t+1} \int_{t}^{u} y\left(s^{-}\right) d s\right]+\mathbb{E}\left[\sup _{t \leq u \leq t+1} \int_{t}^{u} y\left(s^{-}\right) d \tilde{N}(s)\right] \\
& \leq \lambda \mathbb{E} \int_{t}^{t+1} y(s) d s+C_{\lambda} \mathbb{E}\left[\int_{t}^{t+1} y^{2}(s) d s\right]^{1 / 2} \\
& \leq\left(\lambda+C_{\lambda}\right)\left(\mathbb{E} \int_{t}^{t+1} y^{\gamma+1}(s) d s\right)^{1 /(\gamma+1)} .
\end{aligned}
$$


Inserting 4.15) and 4.16 into 4.14, we get

$$
\begin{aligned}
\mathbb{E}\left[\sup _{t \leq u \leq t+1} y(u)\right] \leq & \mathbb{E} y(t)+K_{10}+a_{-1} \int_{t}^{t+1} \mathbb{E} x^{-1}(s) d s+3 b\left(\mathbb{E} \int_{t}^{t+1} y^{\gamma+1}(s) d s\right)^{\theta /(\gamma+1)} \\
& +\delta\left(\lambda+C_{\lambda}\right)\left(\mathbb{E} \int_{t}^{t+1} y^{\gamma+1}(s) d s\right)^{1 /(\gamma+1)} .
\end{aligned}
$$

Note that $1<\theta<(\gamma+1) / 2$ and $1<\gamma \leq 2$. Using the boundedness of $\mathbb{E} y(t), \mathbb{E} y^{-1}(t)$ and $\mathbb{E} y^{2}(t)$, combining with (4.12), we can deduce from 4.17) that there is a constant $K_{11}$ such that

$$
\mathbb{E}\left[\sup _{t \leq u \leq t+1} y(u)\right] \leq K_{11} \text {. }
$$

Let $\varepsilon>0$ be arbitrary. Applying the Chebyshev inequality gives

$$
\mathbb{P}\left(\sup _{n \leq t \leq n+1} y(t)>n^{1+\varepsilon}\right) \leq \frac{K_{11}}{n^{1+\varepsilon}}, \quad n=1,2, \cdots .
$$

By the Borel-Cantelli lemma, we have that for almost $\omega \in \Omega$, there is a $n_{0}(\omega)$, such that

$$
\sup _{n \leq t \leq n+1} y(t) \leq n^{1+\varepsilon}, \quad \text { for } \quad n \geq n_{0}
$$

which means

$$
\log y(t) \leq(1+\varepsilon) \log n \leq(1+\varepsilon) \log t, \quad \text { for } \quad n \leq t \leq n+1 .
$$

Hence,

$$
\limsup _{t \rightarrow \infty} \frac{\log y(t)}{\log t} \leq 1+\varepsilon
$$

Letting $\varepsilon \rightarrow 0$, we obtain the desired assertion 4.9 .

\section{The convergence analysis of the EM method}

In this section, we propose an explicit EM scheme to solve $(1.2)$ and prove a convergence result. We first define a discrete EM scheme to (1.2). For a given fixed time step $\Delta \in(0,1)$ and $Y(0)=y_{0}>0$, the discrete EM approximate solution is defined as below

$$
Y_{n+1}=Y_{n}+f\left(Y_{n}\right) \Delta+b\left|Y_{n}\right|^{\theta} \Delta B_{n}+\delta \Delta Y_{n} \Delta N_{n},
$$

for $n=0,1,2, \cdots$, where $t_{n}=n \Delta, \Delta B_{n}=B\left(t_{n+1}\right)-B\left(t_{n}\right), \Delta N_{n}=N\left(t_{n+1}\right)-N\left(t_{n}\right)$ and $f(y)=$ $a_{-1} y^{-1}-a_{0}+a_{1} y-a_{2} y^{\gamma}$. It is convenient to use the continuous approximation

$$
Y(t)=Y(0)+\int_{0}^{t} f(\bar{Y}(s)) d s+b \int_{0}^{t}|\bar{Y}(s)|^{\theta} d B(s)+\delta \int_{0}^{t} \bar{Y}\left(s^{-}\right) d N(s),
$$

for $t \in[0, T]$, where $\bar{Y}(t)$ is the following step function

$$
\bar{Y}(s)=Y_{n}, \text { for } t \in\left[t_{n}, t_{n+1}\right) .
$$

100 It is easy to see that $\bar{Y}(s)=Y\left(t_{n}\right)=Y_{n}$, for $t \in\left[t_{n}, t_{n+1}\right)$, that is, the discrete and continuous EM solutions coincide at the grid points. The following result generalises Theorem 5.1 in [16] and Theorem 5.4 in [18]. 
Theorem 5.1. For any $T>0$,

$$
\lim _{\Delta \rightarrow 0}\left(\sup _{0 \leq t \leq T}|y(t)-Y(t)|^{2}\right)=0 \text { in probability. }
$$

Proof. We divide the proof into three steps.

Step 1. For any sufficiently large positive integer $n$, we define the stopping time

$$
v_{n}=\inf \{t \in[0, T]: Y(t) \notin[1 / n, n]\} .
$$

Let $V$ be the same as before. Then, for $t \in[0, T]$, Lemma 2.1 gives

$$
\begin{aligned}
\mathbb{E} V\left(Y\left(t \wedge v_{n}\right)\right)= & V\left(Y_{0}\right)+\mathbb{E} \int_{0}^{t \wedge v_{n}}\left[V^{\prime}(Y(s)) f(\bar{Y}(s))+\frac{b^{2}}{2} V^{\prime \prime}(Y(s))|\bar{Y}(s)|^{2 \theta}\right] d s \\
& +\lambda \mathbb{E} \int_{0}^{t \wedge v_{n}}\left[V\left(Y\left(s^{-}\right)+\delta \bar{Y}\left(s^{-}\right)\right)-V\left(Y\left(s^{-}\right)\right)\right] d s .
\end{aligned}
$$

Recalling 2.1) and 2.2, we have that for $s \in\left[0, t \wedge v_{n}\right]$,

$$
\begin{aligned}
& V^{\prime}(Y(s)) f(\bar{Y}(s))+\frac{b^{2}}{2} V^{\prime \prime}(Y(s))|\bar{Y}(s)|^{2 \theta}+\lambda\left[V\left(Y\left(s^{-}\right)+\delta \bar{Y}\left(s^{-}\right)\right)-V\left(Y\left(s^{-}\right)\right)\right] \\
& =V^{\prime}(Y(s)) f(Y(s))+\frac{b^{2}}{2} V^{\prime \prime}(Y(s))|Y(s)|^{2 \theta}+\lambda\left[V\left(Y\left(s^{-}\right)+\delta Y\left(s^{-}\right)\right)-V\left(Y\left(s^{-}\right)\right)\right] \\
& \quad+V^{\prime}(Y(s))(f(\bar{Y}(s))-f(Y(s)))+\frac{b^{2}}{2} V^{\prime \prime}(Y(s))\left(|\bar{Y}(s)|^{2 \theta}-|Y(s)|^{2 \theta}\right) \\
& \quad+\lambda\left[V\left(Y\left(s^{-}\right)+\delta \bar{Y}\left(s^{-}\right)\right)-V\left(Y\left(s^{-}\right)+\delta Y\left(s^{-}\right)\right)\right] \\
& \leq K_{1}+I_{1}(s)+I_{2}(s)+I_{3}\left(s^{-}\right)
\end{aligned}
$$

where

$$
\begin{aligned}
I_{1}(s) & =V^{\prime}(Y(s))(f(\bar{Y}(s))-f(Y(s))), \\
I_{2}(s) & =\frac{b^{2}}{2} V^{\prime \prime}(Y(s))\left(|\bar{Y}(s)|^{2 \theta}-|Y(s)|^{2 \theta}\right), \\
I_{3}\left(s^{-}\right) & =\lambda\left[V\left(Y\left(s^{-}\right)+\delta \bar{Y}\left(s^{-}\right)\right)-V\left(Y\left(s^{-}\right)+\delta Y\left(s^{-}\right)\right)\right] .
\end{aligned}
$$

If $s \in\left[0, t \wedge v_{n}\right]$, then $Y\left(s^{-}\right) \in[1 / n, n]$, which implies $\bar{Y}(s) \in[1 / n, n]$, we can deduce that there are constants $C_{1}(n)$ and $C_{2}(n)$ such that

$$
\begin{aligned}
\left|Y^{2 \theta}(s)-\bar{Y}^{2 \theta}(s)\right| & \leq C_{1}(n)|Y(s)-\bar{Y}(s)|^{2 \theta} \\
& \leq C_{1}(n)|Y(s)-\bar{Y}(s)|(|Y(s)|+|\bar{Y}(s)|)^{2 \theta-1} \\
& \leq C_{1}(n)(2 n)^{2 \theta-1}|Y(s)-\bar{Y}(s)|
\end{aligned}
$$

and

$$
\left|Y^{\gamma}(s)-\bar{Y}^{\gamma}(s)\right| \leq C_{2}(n)|Y(s)-\bar{Y}(s)|
$$


Hence, for any $s \in\left[0, t \wedge v_{n}\right]$, we obtain

$$
\begin{aligned}
I_{1}(s) & =V^{\prime}(Y(s))(f(\bar{Y}(s))-f(Y(s))) \\
& \leq \alpha Y^{-1}(s)\left(Y^{\alpha}(s)-1\right)\left(a_{-1}\left|\frac{1}{\bar{Y}(s)}-\frac{1}{Y(s)}\right|+a_{1}|\bar{Y}(s)-Y(s)|+a_{2}\left|\bar{Y}^{r}(s)-Y^{r}(s)\right|\right) \\
& \leq \alpha n\left(n^{\alpha}-1\right)\left(a_{-1} \frac{|Y(s)-\bar{Y}(s)|}{|Y(s)| \bar{Y}(s) \mid}+a_{1}|Y(s)-\bar{Y}(s)|+a_{2} C_{2}(n)|Y(s)-\bar{Y}(s)|\right) \\
& \leq \alpha n\left(n^{\alpha}-1\right)\left(a_{-1} n^{2}+a_{1}+a_{2} C_{2}(n)\right)|Y(s)-\bar{Y}(s)| \\
& =: C_{3}(n)|Y(s)-\bar{Y}(s)| .
\end{aligned}
$$

Similarly, for any $s \in\left[0, t \wedge v_{n}\right]$, 5.7) gives

$$
\begin{aligned}
I_{2}(s) & =\frac{b^{2}}{2} V^{\prime \prime}(Y(s))\left(\bar{Y}^{2 \theta}(s)-Y^{2 \theta}(s)\right) \\
& \leq \frac{b^{2}}{2}\left|\alpha(\alpha-1) Y^{\alpha-2}(s)+\alpha \bar{Y}^{-2}(s)\right| C_{1}(n)(2 n)^{2 \theta-1}|Y(s)-\bar{Y}(s)| \\
& \leq \frac{b^{2}}{2}(2 n)^{2 \theta-1} C_{1}(n)\left(\alpha(\alpha-1) n^{2-\alpha}+\alpha n^{2}\right)|Y(s)-\bar{Y}(s)| \\
& =: C_{4}(n)|Y(s)-\bar{Y}(s)| .
\end{aligned}
$$

Note that the functions $y^{\alpha}$ and $\log y$ are locally Lipschitz continuous for any $y>0$. Therefore, for $Y(s), \bar{Y}(s) \in[1 / n, n]$, we have

$$
\begin{aligned}
I_{3}\left(s^{-}\right)= & \lambda\left(V\left(Y\left(s^{-}\right)+\delta \bar{Y}\left(s^{-}\right)\right)\right)-V\left(Y\left(s^{-}\right)+\delta Y\left(s^{-}\right)\right) \\
\leq & \lambda\left(\left|\left(Y\left(s^{-}\right)+\delta \bar{Y}\left(s^{-}\right)\right)^{\alpha}-\left(Y\left(s^{-}\right)+\delta Y\left(s^{-}\right)\right)^{\alpha}\right|\right. \\
& \left.+\alpha\left|\log \left(Y\left(s^{-}\right)+\delta \bar{Y}\left(s^{-}\right)\right)^{\alpha}-\log \left(Y\left(s^{-}\right)+\delta Y\left(s^{-}\right)\right)^{\alpha}\right|\right) \\
\leq & \lambda\left(\alpha n^{1-\alpha} \delta\left|Y\left(s^{-}\right)-\bar{Y}\left(s^{-}\right)\right|+n \delta\left|Y\left(s^{-}\right)-\bar{Y}\left(s^{-}\right)\right|\right) \\
= & \lambda \delta\left(\alpha n^{1-\alpha}+n\right)\left|Y\left(s^{-}\right)-\bar{Y}\left(s^{-}\right)\right| \\
= & : C_{5}(n)\left|Y\left(s^{-}\right)-\bar{Y}\left(s^{-}\right)\right| .
\end{aligned}
$$

Inserting 5.9, 5.10) and 5.11 into 5.6, we get from 5.5) that

$$
\left.\mathbb{E} V\left(Y\left(t \wedge v_{n}\right)\right) \leq V\left(y_{0}\right)\right)+K_{1} T+\left(C_{3}(n)+C_{4}(n)+C_{5}(n)\right) \mathbb{E} \int_{0}^{t \wedge v_{n}}|Y(s)-\bar{Y}(s)| d s .
$$

For any $s \in\left[0, T \wedge v_{n}\right]$, there is a constant $C_{6}(n)$ such that $\left|f\left(Y_{\lfloor s / \Delta\rfloor}\right)\right| \leq C_{6}(n)$. By definition [5.1, we have

$$
\begin{aligned}
Y(s)-\bar{Y}(s)= & \left.f\left(Y_{\lfloor s / \Delta\rfloor}\right)(s-\lfloor s / \Delta\rfloor \Delta)\right)+b\left|Y_{\lfloor s / \Delta\rfloor}\right|^{\theta}\left(B(s)-B\left(Y_{\lfloor s / \Delta\rfloor}\right)\right) \\
& +\delta Y_{\lfloor s / \Delta\rfloor}\left(N(s)-N\left(Y_{\lfloor s / \Delta\rfloor}\right)\right) \\
\leq & C_{6}(n) \Delta+b n^{\theta}\left(B(s)-B\left(Y_{\lfloor s / \Delta\rfloor}\right)\right)+\delta n\left(N(s)-N\left(Y_{\lfloor s / \Delta\rfloor}\right)\right) .
\end{aligned}
$$


Hence, for $\Delta \in(0,1)$, we have

$$
\begin{aligned}
\mathbb{E} \int_{0}^{t \wedge v_{n}}|Y(s)-\bar{Y}(s)| d s & \leq C_{6}(n) T \Delta+b n^{\theta} T \Delta^{1 / 2}+\delta n T \lambda \Delta \\
& \leq T\left(C_{6}(n)+b n^{\theta}+\delta n \lambda\right) \Delta^{1 / 2} \\
& =: C_{7}(n) \Delta^{1 / 2} .
\end{aligned}
$$

Substituting this into 5.12 yields

$$
\mathbb{E} V\left(Y\left(t \wedge v_{n}\right)\right) \leq V\left(y_{0}\right)+K_{1} T+C(n) \Delta^{1 / 2},
$$

where $C(n)=\left(C_{3}(n)+C_{4}(n)+C_{5}(n)\right) C_{7}(n)$. Hence,

$$
\mathbb{P}\left(v_{n} \leq T\right) \leq \frac{V\left(y_{0}\right)+K_{1} T+C(n) \Delta^{1 / 2}}{V(1 / n) \wedge V(n)} .
$$

Step 2. Let $\sigma_{n}=\tau_{n} \wedge v_{n}$. We next show that there exists a constant $D(n)$, which is dependent of $n$, such that

$$
\mathbb{E}\left[\sup _{0 \leq t \leq T \wedge \sigma_{n}}|y(t)-Y(t)|^{2}\right] \leq D(n) \Delta .
$$

Note that the function $y^{\theta}$ is locally Lipschitz continuous for $y>0$ and $\theta>1$. Therefore, for $y(s)$, $\bar{Y}(s) \in[1 / n, n]$, there is a constant $C_{8}(n)$ such that

$$
\left|y^{\theta}(s)-\bar{Y}^{\theta}(s)\right|^{2} \leq C_{8}(n)|y(s)-\bar{Y}(s)|^{2} .
$$

For any $t \in[0, T]$ and $s \in\left[0, t \wedge \sigma_{n}\right]$, which implies $x(s), X(s) \in[1 / n, n]$, we have

$$
\begin{aligned}
|f(y(s))-f(\bar{Y}(s))| & \leq a_{-1}\left|\frac{1}{y(s)}-\frac{1}{\bar{Y}(s)}\right|+a_{1}|y(s)-\bar{Y}(s)|+a_{2}\left|y^{2}(s)-\bar{Y}^{2}(s)\right| \\
& \leq a_{-1} \frac{|y(s)-\bar{Y}(s)|}{|y(s)||\bar{Y}(s)|}+a_{1}|y(s)-\bar{Y}(s)|+a_{2}(|y(s)|+|\bar{Y}(s)|)|y(s)-\bar{Y}(s)| \\
& \leq\left(a_{-1} n^{2}+a_{1}+2 a_{2} n\right)|y(s)-\bar{Y}(s)| .
\end{aligned}
$$

By (1.2) and 5.2, we get

$$
\begin{aligned}
y\left(t_{1} \wedge \sigma_{n}\right)-Y\left(t_{1} \wedge \sigma_{n}\right)= & \int_{0}^{t_{1} \wedge \sigma_{n}}(f(y(s))-f(\bar{Y}(s))) d s+b \int_{0}^{t_{1} \wedge \sigma_{n}}\left(y^{\theta}(s)-|\bar{Y}(s)|^{\theta}\right) d B(s) \\
& +\delta \int_{0}^{t_{1} \wedge \sigma_{n}}\left(y\left(s^{-}\right)-\bar{Y}\left(s^{-}\right)\right) d N(s)
\end{aligned}
$$


for any $t_{1} \in[0, T]$. Hence, for any $T \in[0, T]$, by the Hölder and the Doob martingale inequality as well as Lemma 2.2, we have

$$
\begin{aligned}
\mathbb{E} & \left.\sup _{0 \leq t_{1} \leq t}\left|y\left(t_{1} \wedge \sigma_{n}\right)-Y\left(t_{1} \wedge \sigma_{n}\right)\right|^{2}\right] \\
\leq & 3 t \mathbb{E} \int_{0}^{t \wedge \sigma_{n}}(f(y(s))-f(\bar{Y}(s)))^{2} d s+3 b^{2} \mathbb{E}\left[\sup _{0 \leq t_{1} \leq t}\left(\int_{0}^{t_{1} \wedge \sigma_{n}}\left(y^{\theta}(s)-|\bar{Y}(s)|^{\theta}\right) d B(s)\right)^{2}\right] \\
& +3 \delta^{2} \mathbb{E}\left[\sup _{0 \leq t_{1} \leq t}\left(\int_{0}^{t_{1} \wedge \sigma_{n}}\left(y\left(s^{-}\right)-\bar{Y}\left(s^{-}\right)\right) d N(s)\right)^{2}\right] \\
\leq & 3 t\left(a_{-1} n^{2}+a_{1}+2 a_{2} n\right)^{2} \mathbb{E}\left[\int_{0}^{t \wedge \sigma_{n}}|y(s)-\bar{Y}(s)|^{2} d s\right]+12 b^{2} \mathbb{E}\left[\int_{0}^{t \wedge \sigma_{n}}\left(y^{\theta}(s)-|\bar{Y}(s)|^{\theta}\right)^{2} d s\right] \\
& +3 \delta^{2} \lambda^{2} \mathbb{E}\left[\int_{0}^{t \wedge \sigma_{n}}(y(s)-\bar{Y}(s)) d s\right]^{2}+K \mathbb{E}\left[\int_{0}^{t \wedge \sigma_{n}}(y(s)-\bar{Y}(s))^{2} d s\right] \\
\leq & {\left[3 t\left(a_{-1} n^{2}+a_{1}+2 a_{2} n\right)^{2}+12 b^{2} C_{8}(n)+2 \delta^{3} \lambda^{2} t+K\right] \mathbb{E}\left[\int_{0}^{t \wedge \sigma_{n}}(y(s)-\bar{Y}(s))^{2} d s\right] } \\
= & : C_{9}(n) \mathbb{E}\left[\int_{0}^{t \wedge \sigma_{n}}(y(s)-\bar{Y}(s))^{2} d s\right] \\
\leq & 2 C_{9}(n) \int_{0}^{t} \mathbb{E}\left(y\left(s \wedge \sigma_{n}\right)-Y\left(s \wedge \sigma_{n}\right)\right)^{2} d s++2 C_{9}(n) \mathbb{E}\left[\int_{0}^{t \wedge \sigma_{n}}(Y(s)-\bar{Y}(s))^{2} d s\right],
\end{aligned}
$$

where $K$ is a constant. In the same way as the computation of (5.14), we can see that there exists a constant $C_{10}(n)$ such that

$$
\mathbb{E} \int_{0}^{t \wedge \sigma_{n}}(Y(s)-\bar{Y}(s))^{2} d s \leq C_{10}(n) \Delta .
$$

Inserting this into (5.21) gives

$$
\begin{aligned}
& \mathbb{E}\left[\sup _{0 \leq t_{1} \leq t}\left|y\left(t_{1} \wedge \sigma_{n}\right)-Y\left(t_{1} \wedge \sigma_{n}\right)\right|^{2}\right] \\
& \leq 2 C_{9}(n) \int_{0}^{t} \mathbb{E}\left(y\left(s \wedge \sigma_{n}\right)-Y\left(s \wedge \sigma_{n}\right)\right)^{2} d s+2 C_{9}(n) C_{10}(n) \Delta .
\end{aligned}
$$

Applying the Gronwall inequality yields 5.17).

Step 3. Let $\varepsilon_{1}, \varepsilon_{2} \in(0,1)$ be arbitrarily small. Set

$$
\bar{\Omega}=\left\{\omega: \sup _{0 \leq t \leq T}|y(t)-Y(t)|^{2} \geq \varepsilon_{1}\right\} .
$$

Using [5.17), we have

$$
\begin{aligned}
\varepsilon_{1} \mathbb{P}\left(\bar{\Omega} \cap\left\{\sigma_{n} \geq T\right\}\right) & \leq \mathbb{E}\left[\mathbb{I}_{\left\{\sigma_{n} \geq T\right\}} \mathbb{I}_{\bar{\Omega}}\right] \\
& \leq \mathbb{E}\left[\mathbb{I}_{\left\{\sigma_{n} \geq T\right\}} \sup _{0 \leq t \leq T \wedge \sigma_{n}}|y(t)-Y(t)|^{2}\right] \\
& \leq \mathbb{E}\left[\sup _{0 \leq t \leq T \wedge \sigma_{n}}|y(t)-Y(t)|^{2}\right] \\
& \leq D(n) \Delta .
\end{aligned}
$$


By [2.4), 5.16) and 5.22, we have

$$
\begin{aligned}
\mathbb{P}(\bar{\Omega}) & \leq \mathbb{P}\left(\bar{\Omega} \cap\left\{\sigma_{n} \geq T\right\}\right)+\mathbb{P}\left(\sigma_{n} \leq T\right) \\
& \leq \mathbb{P}\left(\bar{\Omega} \cap\left\{\sigma_{n} \geq T\right\}\right)+\mathbb{P}\left(\tau_{n} \leq T\right)+\mathbb{P}\left(v_{n} \leq T\right) \\
& \leq \frac{D(n)}{\varepsilon_{1}} \Delta+\frac{2 V\left(y_{0}\right)+2 K_{1} T+c(n) \Delta^{1 / 2}}{V(1 / n) \wedge V(n)} .
\end{aligned}
$$

Recalling that $V(1 / n) \wedge V(n) \rightarrow \infty$, as $n \rightarrow \infty$, we can choose $n$ sufficiently large for

$$
\frac{2 V\left(y_{0}\right)+2 K_{1} T}{V(1 / n) \wedge V(n)} \leq \frac{\varepsilon_{2}}{2}
$$

and then choose $\Delta$ sufficiently small for

$$
\frac{D(n)}{\varepsilon_{1}} \Delta+\frac{c(n) \Delta^{1 / 2}}{V(1 / n) \wedge V(n)} \leq \frac{\varepsilon_{2}}{2}
$$

to obtain

$$
\mathbb{P}(\bar{\Omega})=\mathbb{P}\left(\sup _{0 \leq t \leq T}|y(t)-Y(t)|^{2} \geq \varepsilon_{1}\right) \leq \varepsilon_{2},
$$

which is the desired assertion (5.4).

Lemma 5.2. For any $T>0$,

$$
\lim _{\Delta \rightarrow 0}\left(\sup _{0 \leq t \leq T}|Y(t)-\bar{Y}(t)|^{2}\right)=0 \quad \text { in probability. }
$$

Proof. By [5.13, for $t \in\left[0, T \wedge v_{n}\right]$, we have

$$
\begin{aligned}
\mathbb{E}\left[\sup _{0 \leq t \leq T \wedge v_{n}}|Y(t)-\bar{Y}(t)|^{2}\right] \leq & C_{11}(n) \Delta^{2}+C_{12}(n) \mathbb{E}\left[\sup _{0 \leq t \leq T \wedge v_{n}}|B(t)-B(\lfloor t / \Delta\rfloor \Delta)|^{2}\right] \\
& +C_{13}(n) \mathbb{E}\left[\sup _{0 \leq t \leq T \wedge v_{n}}|\tilde{N}(t)-\tilde{N}(\lfloor t / \Delta\rfloor \Delta)|^{2}\right],
\end{aligned}
$$

where $C_{11}(n)=3 C_{6}^{2}(n)+6 \lambda^{2} \delta^{2} n^{2}, C_{12}(n)=3 b^{2} n^{2 \theta}$ and $C_{13}(n)=6 \delta^{2} n^{2}$. The Doob martingale inequality gives

$$
\begin{aligned}
\mathbb{E}\left[\sup _{0 \leq t \leq T}|B(t)-B(\lfloor t / \Delta\rfloor \Delta)|^{4}\right] & =\mathbb{E}\left[\max _{0 \leq n \leq L T / \Delta\rfloor-1} \sup _{n \Delta \leq t \leq(n+1) \Delta}|B(t)-B(n \Delta)|^{4}\right] \\
& \leq \sum_{n=0}^{\lfloor T / \Delta\rfloor-1} \mathbb{E}\left[\sup _{n \Delta \leq t \leq(n+1) \Delta}|B(t)-B(n \Delta)|^{4}\right] \\
& \leq(4 / 3)^{4} \sum_{n=0}^{[T / \Delta\rfloor-1} \mathbb{E}\left[|B((n+1) \Delta)-B(n \Delta)|^{4}\right] \\
& \leq \frac{256}{27} \sum_{n=0}^{[T / \Delta\rfloor-1} \Delta^{2} \\
& \leq \frac{256}{27} T \Delta .
\end{aligned}
$$


By the Hölder inequality, we get

$$
\begin{aligned}
\mathbb{E}\left[\sup _{0 \leq t \leq T}|B(t)-B(\lfloor t / \Delta\rfloor \Delta)|^{2}\right] & \leq\left(\mathbb{E}\left[\sup _{0 \leq t \leq T}|B(t)-B(\lfloor t / \Delta\rfloor \Delta)|^{4}\right]\right)^{1 / 2} \\
& \leq \frac{16}{27^{1 / 2}} T \Delta^{1 / 2}
\end{aligned}
$$

Note that $N(T)$ is a Poisson process with intensity $\lambda$, which means $\mathbb{P}(N(T)<\infty)=1$, we let these jump points within the intervals $\left[k_{1} \Delta,\left(k_{1}+1\right) \Delta\right),\left[k_{2} \Delta,\left(k_{2}+1\right) \Delta\right), \cdots,\left[k_{N(T)} \Delta,\left(k_{N(T)}+1\right) \Delta\right)$, respectively. Hence, by the Doob martingale inequality, we have

$$
\begin{aligned}
\mathbb{E}\left[\sup _{0 \leq t \leq T}\left|\tilde{N}\left(t^{-}\right)-\tilde{N}\left(\lfloor t / \Delta\rfloor \Delta^{-}\right)\right|^{2}\right] & \leq \mathbb{E}\left[\max _{1 \leq i \leq N(T)} \sup _{k_{i} \Delta \leq t<\left(k_{i}+1\right) \Delta}\left|\tilde{N}\left(t^{-}\right)-\tilde{N}\left(k_{i} \Delta^{-}\right)\right|^{2}\right] \\
& \leq \mathbb{E}\left[\sum_{i=1}^{N(T)} \sup _{k_{i} \Delta \leq t<\left(k_{i}+1\right) \Delta}\left|\tilde{N}\left(t^{-}\right)-\tilde{N}\left(k_{i} \Delta^{-}\right)\right|^{2}\right] \\
& \leq \mathbb{E} N(T) \mathbb{E}\left[\sup _{k_{i} \Delta \leq t<\left(k_{i}+1\right) \Delta}\left|\tilde{N}\left(t^{-}\right)-\tilde{N}\left(k_{i} \Delta^{-}\right)\right|^{2}\right] \\
& \leq 4 \mathbb{E} N(T) \mathbb{E}\left[\left|\tilde{N}\left(\left(k_{i}+1\right) \Delta^{-}\right)-\tilde{N}\left(k_{i} \Delta^{-}\right)\right|^{2}\right] \\
& \leq C_{14} \Delta,
\end{aligned}
$$

where $C_{14}$ is a positive constant. Substituting (5.27) and 5.28 into 5.26 gives that there is a constant $C_{15}(n)$ such that

$$
\mathbb{E}\left[\sup _{0 \leq t \leq T \wedge v_{n}}|Y(t)-\bar{Y}(t)|^{2}\right] \leq C_{15}(n) \Delta^{1 / 2} .
$$

Let $\varepsilon_{1}, \varepsilon_{2} \in(0,1)$ be arbitrary small, we define

$$
\tilde{\Omega}=\left\{\omega: \sup _{0 \leq t \leq T}|Y(t)-\bar{Y}(t)|^{2} \geq \varepsilon_{1}\right\} .
$$

Then, we have

$$
\begin{aligned}
\varepsilon_{1} \mathbb{P}\left(\tilde{\Omega} \cap\left\{v_{n} \geq T\right\}\right) & \leq \mathbb{E}\left[\mathbb{I}_{\left\{v_{n} \geq T\right\}} \mathbb{I}_{\tilde{\Omega}}\right] \\
& \leq \mathbb{E}\left[\mathbb{I}_{\left\{v_{n} \geq T\right\}} \sup _{0 \leq t \leq T \wedge v_{n}}|Y(t)-\bar{Y}(t)|^{2}\right] \\
& \leq \mathbb{E}\left[\sup _{0 \leq t \leq T \wedge v_{n}}|Y(t)-\bar{Y}(t)|^{2}\right] \\
& \leq C_{15}(n) \Delta^{1 / 2} .
\end{aligned}
$$

Thus, by $[5.30)$ and 5.16$]$, we have

$$
\begin{aligned}
\mathbb{P}(\tilde{\Omega}) & \leq \mathbb{P}\left(\tilde{\Omega} \cap\left\{v_{n} \geq T\right\}\right)+\mathbb{P}\left(v_{n} \leq T\right) \\
& \leq \frac{C_{15}(n)}{\varepsilon_{1}} \Delta^{1 / 2}+\frac{V\left(y_{0}\right)+K_{1} T+c(n) \Delta^{1 / 2}}{V(1 / n) \wedge V(n)} .
\end{aligned}
$$


Recalling that $V(1 / n) \wedge V(n) \rightarrow \infty$, as $n \rightarrow \infty$, we can choose $n$ sufficiently large for

$$
\frac{V\left(x_{0}\right)+K_{1} T}{V(1 / n) \wedge V(n)} \leq \frac{\varepsilon_{2}}{2}
$$

and then choose $\Delta$ sufficiently small for

$$
\frac{C_{15}(n)}{\varepsilon_{1}} \Delta^{1 / 2}+\frac{c(n) \Delta^{1 / 2}}{V(1 / n) \wedge V(n)} \leq \frac{\varepsilon_{2}}{2}
$$

to obtain

$$
\mathbb{P}(\tilde{\Omega})=\mathbb{P}\left(\sup _{0 \leq t \leq T}|Y(t)-\bar{Y}(t)|^{2} \geq \varepsilon_{1}\right) \leq \varepsilon_{2} .
$$

Thus, we complete the proof

Theorem 5.3. For any $T>0$,

$$
\lim _{\Delta \rightarrow 0}\left(\sup _{0 \leq t \leq T}|y(t)-\bar{Y}(t)|^{2}\right)=0 \quad \text { in probability. }
$$

Proof. For all sufficiently small $\varepsilon \in(0,1)$,

$$
\mathbb{P}\left(\sup _{0 \leq t \leq T}|y(t)-\bar{Y}(t)| \geq \varepsilon\right) \leq \mathbb{P}\left(\sup _{0 \leq t \leq T}|y(t)-Y(t)| \geq \varepsilon / 2\right)+\mathbb{P}\left(\sup _{0 \leq t \leq T}|Y(t)-\bar{Y}(t)| \geq \varepsilon / 2\right) .
$$

By Theorem 5.1 and Lemma 5.2 , we obtain the desired assertion.

Remark 5.4. It should be pointed out that when Poisson process $N(t)$ is ignored in the model 110 (1.2), the results on the analytical properties of the solution to the model and convergence of the numerical method reduce to the corresponding ones that were obtained in [10], [16] and [18]. We extend these results to the case of Poisson jumps.

\section{Applications in finance}

In this section, we assume that the interest rate or asset price is governed by the model 1.2 .

Then we use the EM method to approximate some financial quantities.

\subsection{Bonds}

Let $y(t)$ be the short-term interest rate. Denote by $B(T)$ the price of a bond at the end of period with form

$$
B(T)=\mathbb{E}\left[\exp \left(-\int_{0}^{T} y(t) d t\right)\right]
$$

An approximation to $B(T)$ is given by

$$
\bar{B}_{\Delta}(T)=\mathbb{E}\left[\exp \left(-\int_{0}^{T}|\bar{Y}(t)| d t\right)\right],
$$


where $\bar{Y}(t)$ is defined in (5.3). Then we have

$$
\lim _{\Delta \rightarrow 0}\left|B(T)-\bar{B}_{\Delta}(T)\right|=0 .
$$

Let $\varepsilon, \xi \in(0,1)$ be arbitrarily small. We need to prove

$$
\mathbb{P}\left(\left|\exp \left(-\int_{0}^{T} y(t) d t\right)-\exp \left(-\int_{0}^{T}|\bar{Y}(t)| d t\right)\right| \geq \xi\right)<\varepsilon .
$$

Note that

$$
\begin{aligned}
\left|\exp \left(-\int_{0}^{T} y(t) d t\right)-\exp \left(-\int_{0}^{T}|\bar{Y}(t)| d t\right)\right| & \leq\left|\int_{0}^{T}[y(t)-\bar{Y}(t)] d t\right| \\
& \leq T \sup _{0 \leq t \leq T}|y(t)-\bar{Y}(t)| .
\end{aligned}
$$

Using the Theorem 5.3, we get the desired assertion.

\subsection{Barrier options}

Let $E$ be the exercise price. Denote by $y(T)$ the asset price at the expiry date $T$ and $B$ the fixed barrier. The expected value of the barrier options at the expiry date, denoted by $C$, is defined as below

$$
C(T)=\mathbb{E}\left[(y(T)-E)^{+} \mathbb{I}_{\{0 \leq y(t) \leq B, 0 \leq t \leq T\}}\right] .
$$

We define its approximation by

$$
\bar{C}_{\Delta}(T)=\mathbb{E}\left[(\bar{Y}(T)-E)^{+} \mathbb{I}_{\{0 \leq \bar{Y}(t) \leq B, 0 \leq t \leq T\}}\right],
$$

where $\bar{Y}(t)$ is the same as before. Then

$$
\lim _{\Delta \rightarrow 0}\left|C(T)-\bar{C}_{\Delta}(T)\right|=0 .
$$

The proof can be found in [10].

The above examples show that the EM method can be used to estimate finance quantities.

\section{A numerical example}

Let us consider the following Ait-Sahalia interest rate model with Poisson jumps

$$
\begin{aligned}
d y(t) & =\left(a_{-1} y^{-1}(t)-a_{0}+a_{1} y(t)-a_{2} y^{\gamma}(t)\right) d t+b y^{\theta}(t) d B(t)+\delta y\left(t^{-}\right) d N(t), \\
y(0) & =y_{0} .
\end{aligned}
$$

We use a part of the parameters that were estimated from financial data (see [13, 41]), namely,

$$
\begin{aligned}
a_{-1} & =1.041 \times 10^{-4}, & a_{0} & =5.652 \times 10^{-3}, \\
a_{1} & =9.648 \times 10^{-2}, & a_{2} & =5.349 \times 10^{-1}, \\
b & =1.329 \times 10^{-2}, & \delta & =1.041 \times 10^{-4}, \\
\gamma & =2, & \theta & =1.4999, \\
y_{0} & =6.0 \%, & \lambda & \lambda=0.20,
\end{aligned}
$$

where $y_{0}$ denotes the initial value for the short-term rate of interest and $\lambda$ the mean number of jumps per unit time. Let $y(t)$ be the true solution of 7.1) and $Y(t)$ the corresponding EM solution defined in 5.2. In this section, sample average is used to approximate the expected value. 
In order to test the moment boundedness of the true solution, we carry out numerical experiments using MATLAB. Fig. 3 shows the values of $\mathbb{E} y(t), \mathbb{E} y^{2}(t)$ and $\mathbb{E} y^{-1}(t)$ versus $t$ for the Ait-Sahalia model 7.1), approximated by EM scheme (5.1) with step size $\Delta=0.005$ and 500 sample paths, respectively. We see from Fig. 3 that sample averages of $Y(t), Y^{2}(t)$ and $Y^{-1}(t)$ are bounded, confirming the results of Theorem 3.1 and 3.2.

\subsection{The pathwise estimations}

We perform a simulation result plotted in Fig.2 on the evolution of $\log Y(t) / \log t$ with time $t$ for 50 solution paths of 7.1. The points have been joined by blue straight lines for clarity. The two curves $1+\varepsilon$ and $-(1+\varepsilon)$ are added as red dotted and black dash-dot lines, respectively. It seems that curves representing $\log Y(t) / \log t$ lie between the two straight lines $-(1+\varepsilon)$ and $1+\varepsilon$ for all the samples if time $t$ is sufficiently large, say, $t \geq 20$. On the other hand, by virtue of Theorems 4.1 and 4.2 we obtain that for any $\varepsilon>0$, there is a random variable $T_{\varepsilon}$ such that the true solution $y(t)$ of (7.1) satisfied

$$
-(1+\varepsilon) \leq \log y(t) / \log t \leq 1+\varepsilon, \text { for } \forall t \geq T_{\varepsilon},
$$

with probability one. Clearly, simulation experiments illustrate the validity of the theoretical results.

\subsection{Convergence}

To show the convergence result, we will focus on the error between the true solution $y(T)$ and EM solution $Y_{L}$ at the endpoint $T$, where $T=L \Delta$. Define the strong error $e_{\Delta}^{\text {strong }}$ by

$$
e_{\Delta}^{\text {strong }}:=\mathbb{E}\left|y(T)-Y_{L}\right| \text {. }
$$

Since it is difficult to obtain an explicit solution to 77.17 , the EM solution with step size $\Delta=2^{-12}$ is used as a reference solution. Fig.2 plots the strong error at endpoint $T=10$ with $\Delta=2^{-i}$ ( $i=5,6,7,8,9)$ on a $\log -\log$ scale for 200 sample paths. Table 1 shows the sample mean of $\left|Y(T)-Y_{L}\right|$ with 200 sample points for different step sizes $\Delta$ and different endpoints $T$. From Table 1 and Fig.2, we deduce that there is a constant $C$ such that $e_{\Delta}^{\text {strong }} \leq C \Delta^{1 / 2}$. Thanks to the Markov inequality, we get

$$
\mathbb{P}\left(\left|y(T)-Y_{L}\right| \geq \Delta^{1 / 4}\right) \leq \frac{\mathbb{E}\left|y(T)-Y_{L}\right|}{\Delta^{1 / 4}} \leq C \Delta^{1 / 4},
$$

which means that the error at a fixed point in $[0, T]$ is small with probability close to one. Thus, the simulation results illustrate the convergence of EM method.

Remark 7.1. Compared with some similar works (e.g., [10, 16, 18, 42]), our numerical experiments show the simulation results on the moment boundedness and the pathwise estimations of the solution to the model as well as the convergence of the numerical scheme for the first time. It is interesting to observe from Fig. 1 that the EM solution is strongly convergent with order onehalf and Fig. 2 that the curves denoting $\log y(t) / \log$ t are far below the straight line $1+\varepsilon$, which is the theoretical estimate for the upper bounds of $\log y(t) / \log t$, for all sample paths. These observations indicate that our theoretical results are somewhat conservative. We will improve this estimate bounds and propose a more appropriate numerical method for solving this model which preserves nonnegativity and converges strongly to the true solution in the future. 
Table 1: Sample mean of $\left|Y(T)-Y_{L}\right|$ with 200 sample points for different step sizes $\Delta$ and different endpoints $T$

\begin{tabular}{cccccc}
\hline$\Delta$ & $2^{-5}$ & $2^{-6}$ & $2^{-7}$ & $2^{-8}$ & $2^{-9}$ \\
\hline$T=10$ & $0.3207 \mathrm{e}-006$ & $0.2093 \mathrm{e}-006$ & $0.1559 \mathrm{e}-006$ & $0.0976 \mathrm{e}-006$ & $0.0632 \mathrm{e}-006$ \\
$T=15$ & $0.3736 \mathrm{e}-006$ & $0.2812 \mathrm{e}-006$ & $0.2007 \mathrm{e}-006$ & $0.1253 \mathrm{e}-002$ & $0.0807 \mathrm{e}-006$ \\
$T=20$ & $0.4190 \mathrm{e}-006$ & $0.2921 \mathrm{e}-006$ & $0.2182 \mathrm{e}-006$ & $0.1519 \mathrm{e}-006$ & $0.0970 \mathrm{e}-006$ \\
$T=25$ & $0.4880 \mathrm{e}-006$ & $0.3672 \mathrm{e}-006$ & $0.2608 \mathrm{e}-006$ & $0.1775 \mathrm{e}-006$ & $0.1137 \mathrm{e}-006$ \\
\hline
\end{tabular}

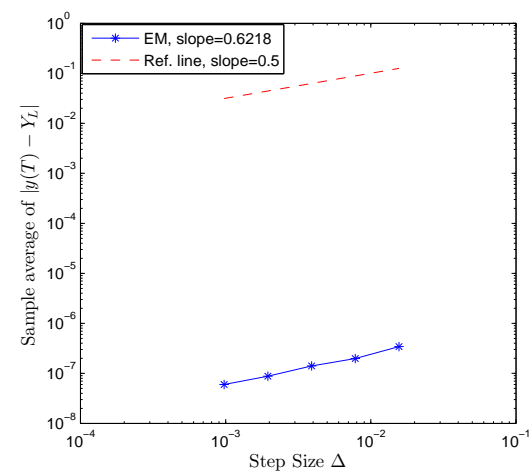

Fig. 1. Strong error by EM at time $T=10$ with 200 sample paths

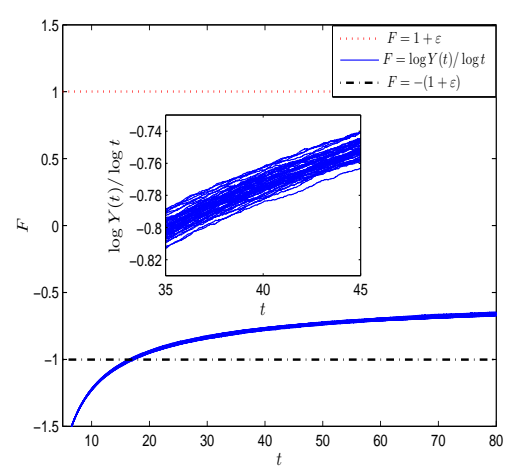

Fig. 2. $\log Y(t) / \log t$ vs. $t$ by EM with $\varepsilon=0.001$ for 50 sample paths

\section{Conclusion}

In this paper, we extend the generalized Ait-Sahalia interest rate model to the case of Poisson jumps. By means of the Lyapunov function method and stochastic analysis technique, the analytical properties of the solution to this model are studied. Also, a convergence result for EM method is obtained. Finally, a simulation example is given to illustrate the effectiveness of our results.

\section{Acknowledgment}

This work was supported by the Natural Science Foundation of China (Nos. 71571001, 61503001, 61703003) and the Major University Science Research Project of Anhui Province (KJ2019ZD16).

\section{References}

[1] A. Balbas, R. Romera, E. Ruiz, Recent Advances in Applied Probability, Springer, Boston, MA, 2005.

[2] R. C. Merton, Theory of rational option pricing, Bell Journal of Economics 4 (1973) 141-183.

[3] O. Vasicek, An equilibrium characterization of the term structure, Journal of Financial and Quantitative Analysis 5 (1977) $177-188$

[4] J. C. Cox, J. E. Ingersoll, S. A. Ross, A theory of the term structure of interest rates, Econometrica 53 (1985) $385-407$. 

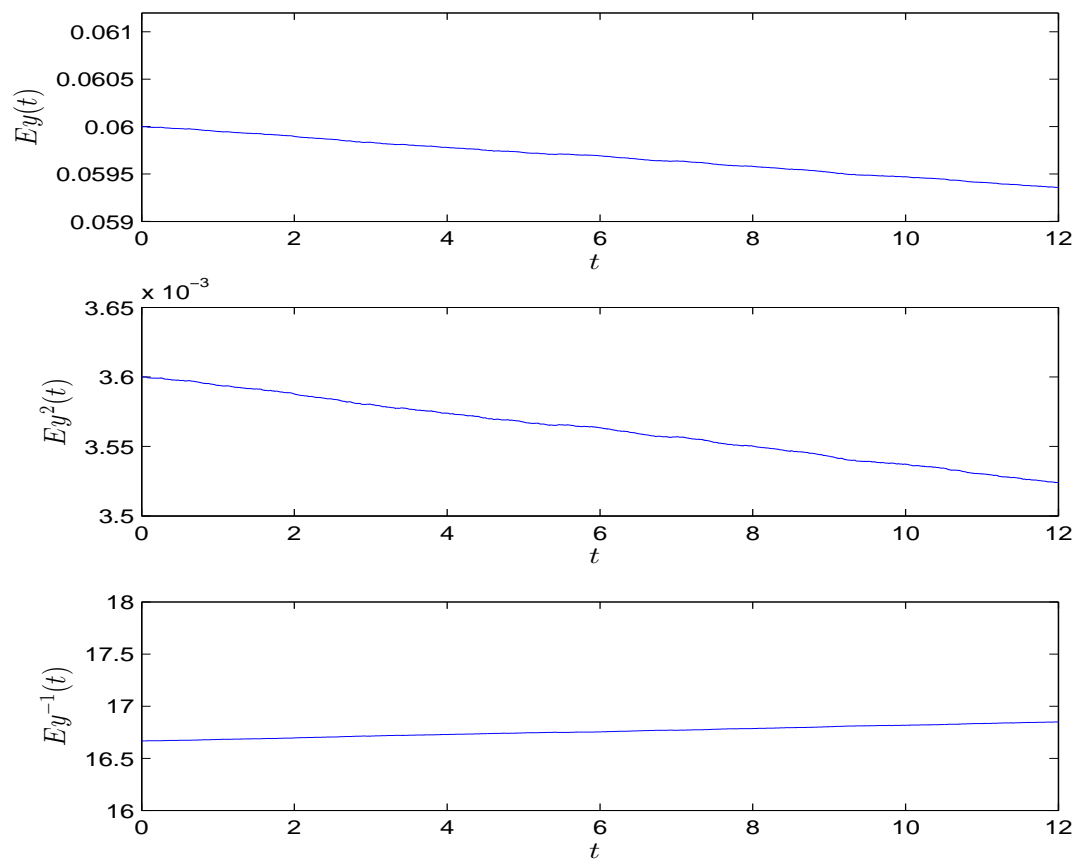

Fig. 3. Sample averages of $Y(t), Y^{2}(t)$ and $Y^{-1}(t)$ by EM with 500 sample paths

[5] M. J. Brennan, E. S. Schwartz, Analyzing convertible bonds, Journal of Financial and Quantitative Analysis 15 (1980) 907-929.

[6] D. Higham, X. Mao, Convergence of Monte Carlo simulations involving the mean-reverting square root process, Journal of Computational Finance 8 (2005) 35-61.

[7] F. Wu, X. Mao, K. Chen, Strong convergence of Monte Carlo simulations of the mean-reverting square root process with jump, Applied Mathematics and Computation 206 (2008) 494-505.

[8] S. Dereich, A. Neuenkirch, L. Szpruch, An Euler-type method for the strong approximation of the Cox-IngersollRoss process, Proceedings: Mathematical, Physical and Engineering Sciences 468 (2012) 1105-1115.

[9] M. Hefter, A. Herzwurm, Strong convergence rates for Cox-Ingersoll-Ross processes-full parameter range, Journal of Mathematical Analysis and Applications 459 (2018) 1079-1101.

[10] F. Wu, X. Mao, K. Chen, A highly sensitive mean-reverting process in finance and the Euler-Maruyama approximations, Journal of Mathematical Analysis and Applications 348 (2008) 540-554.

175 [11] F. Wu, X. Mao, K. Chen, The Cox-Ingersoll-Ross model with delay and strong convergence of its Euler-Maruyama approximate solutions, Applied Numerical Mathematics 59 (2009) 2641-2658.

[12] C. H. Baduraliya, X. Mao, The Euler-Maruyama approximation for the asset price in the mean-reverting-theta stochastic volatility model, Computers and Mathematics with Applications 64 (2012) 2209-2223.

[13] Y. Aït-Sahalia, Testing continuous-time models of the spot interest rate, Review of Financial Studies 9 (1996) $385-426$

[14] Y. Hong, H. Lin, S. Wang, Modeling the dynamics of chinese spot interest rates, Journal of Banking and Finance 34 (2010) 1047-1061.

[15] Z. Lu, Y. Zhu, B. Li, Critical value-based asian option pricing model for uncertain financial markets, Physica A Statistical Mechanics and its Applications 525 (2019) 694-703.

185 [16] S.-R. Cheng, Highly nonlinear model in finance and convergence of Monte Carlo simulations, Journal of Mathematical Analysis and Applications 353 (2009) 531-543. 
[17] L. Szpruch, X. Mao, D. J. Higham, J. Pan, Numerical simulation of a strongly nonlinear Ait-Sahalia-type interest rate model, BIT Numerical Mathematics 51 (2010) 405-425.

[18] F. Jiang, H. Yang, T. Tian, Property and numerical simulation of the Ait-Sahalia-Rho model with nonlinear growth conditions, Discrete and Continuous Dynamical Systems-Series B 22 (2017) 101-113.

[19] X. Jin, Z. Zhang, Ergodicity of generalized Ait-Sahalia-type interest rate model, Communications in StatisticsTheory and Methods 46 (2016) 8199-8209.

[20] M. Dungey, D. Erdemlioglu, M. Matei, X. Yang, Testing for mutually exciting jumps and financial flights in high frequency data, Journal of Econometrics 202 (2018) 18-44.

195 [21] Y. Ait-Sahalia, J. Jacod, Testing for jumps in a discretely observed process, Annals of Statistics 37 (2009) 184-222

[22] Y. Ait-Sahalia, J. Jacod, Estimating the degree of activity of jumps in high frequency data, Annals of Statistics 37 (2009) 2202-2244.

[23] Y. Aït-Sahalia, J. Jacod, J. Li, Testing for jumps in noisy high frequency data, Journal of Econometrics 168 (2012) 207-222.

200 [24] L. Ma, Z. Wang, Q.-L. Han, Y. Liu, Dissipative control for nonlinear Markovian jump systems with actuator failures and mixed time-delays, Automatica 98 (2018) 358-362.

[25] J. Li, H. Dong, Z. Wang, W. Zhang, Protocol-based state estimation for delayed Markovian jumping neural networks, Neural Networks 108 (2018) 355-364.

[26] S. Perera, W. Buckley, H. Long, Market-reaction-adjusted optimal central bank intervention policy in a forex market with jumps, Annals of Operations Research 262 (2018) 213-238.

[27] E. Pillay, J. G. O'Hara, FFT based option pricing under a mean reverting process with stochastic volatility and jumps, Journal of Computational and Applied Mathematics 235 (2011) 3378-3384.

[28] Q. Zhu, Asymptotic stability in the pth moment for stochastic differential equations with Lévy noise, Journal of Mathematical Analysis and Applications 416 (2014) 126-142.

[29] Q. Zhu, Razumikhin-type theorem for stochastic functional differential equations with Lévy noise and markov switching, International Journal of Control 90 (2017) 1703-1712.

[30] L. Liu, Q. Zhu, Almost sure exponential stability of numerical solutions to stochastic delay Hopfield neural networks, Applied Mathematics and Computation 266 (2015) 698-712.

[31] L. Liu, Q. Zhu, Mean square stability of two classes of theta method for neutral stochastic differential delay equations, Journal of Computational and Applied Mathematics 305 (2016) 55-67.

[32] Q. Zhu, Stability analysis of stochastic delay differential equations with Lévy noise, Systems and Control Letters 118 (2018) 62-68.

[33] R. C. Merton, Option pricing when underlying stock returns are discontinuous, Journal of Financial Economics 3 (1976) 125-144.

34] P. Jorion, On jump processes in the foreign exchange and stock markets, Review of Financial Studies 1 (1988) $427-445$.

[35] M. A. Chang, H. E. hompson, Jump-diffusion processes and the term structure of interest rates, journal of Finance 43 (1988) 155-174.

[36] D. S. Bates, Jumps and stochastic volatility: Exchange rate processes implicit in deutsche mark options, Review of Financial Studies 9 (1996) 69-107.

[37] H. Vicky, D. Hobson, Coupling and option price comparisons in a jump-diffusion model, Stochastics and Stochastic Reports 75 (2011) 79-101.

[38] R. Cont, P. Tankov, Financial Modelling with Jump Processes, Chapman and Hall/CRC, 2004.

[39] C. Fei, W. Fei, X. Mao, Delay dependent stability and boundedness of highly nonlinear jump-diffusion stochastic systems, preprint (2018)

[40] X. Mao, Stochastic Differential Equations and Applications, Horwood, 2nd edition, 2008.

[41] K. C. Chan, G. A. Karolyi, F. A. Longstaff, B. S. Anthony, An empirical comparison of alternative models of short-term interest rates, The Journal of Finance 47 (1992) 1209-1227.

[42] F. Jiang, Y. Shen, F. Wu, Jump systems with the mean-reverting gamma-process and convergence of the numerical approximation, Stochastics and Dynamics 12 (2012) 1-15. 